\title{
Profiles of circular RNAs in human blood and their potential roles in preeclampsia
}

Min Liu ${ }^{1}$, Xiaolei Luo ${ }^{1,2}$, Linbo Gao ${ }^{1,2}$, Mengdan Shi ${ }^{1}$, Rong Zhou ${ }^{1}$, Tao Wang ${ }^{1,2}$

${ }^{1}$ Department of Obstetrics and Gynecology, Center for Translational Medicine, Key Laboratory of Birth Defects and Related Diseases of Women and Children (Sichuan University), Ministry of Education, West China Second University Hospital, Sichuan University, Chengdu, China

${ }^{2}$ Center for Translational Medicine, Key Laboratory of Birth Defects and Related Diseases of Women and Children (Sichuan University), Ministry of Education, Department of Obstetrics and Gynecology, West China Second University Hospital, Sichuan University, Chengdu, China

Submitted: 5 September 2021; Accepted: 14 January 2022

Online publication: 18 February 2022

Arch Med Sci

DOI: https://doi.org/10.5114/aoms/145848

Copyright @ 2022 Termedia \& Banach

\section{Abstract}

Introduction: To identify circular RNA (circRNA) expression profiles in the blood of preeclampsia (PE) and healthy pregnant women, and further clarify the possible mechanisms of circRNAs involved in the pathogenesis of PE.

Material and methods: Whole blood samples were collected from 5 paired PE and healthy pregnant women. The differentially expressed circRNAs (DEcircRNAs) were investigated using high-throughput sequencing. Bioinformatics was performed to evaluate the sequencing results and obtain insights into possible mechanisms, such as gene ontology (GO) and KEGG pathway analyses. Then, six DE-circRNAs were chosen and validated by quantitative real-time PCR (RT-qPCR) in an enlarged sample size. Their diagnostic values were analyzed by the receiver operating characteristic (ROC) curve. Establishment and analysis of the circRNA-miRNA-mRNA network were performed for the validated DE-circRNAs.

Results: A total of 139 DE-circRNAs between PE and controls were revealed. Of them, 18 circRNAs were upregulated, and 31 circRNAs were downregulated (fold change $>2$ and $p$-value $<0.05$ ). Three circRNAs (has-circ-0007717, has-circ-0006460, and has-circ-0093055) were higher in both blood of early-onset and late-onset PE patients. The ROC analysis showed that the area under the curve values of has-circ-0007717, has-circ-0006460, and hascirc-0093055 were $0.64(p=0.11), 0.72(p=0.01)$, and $0.72(p=0.01)$, respectively. The circRNA-miRNA-mRNA competing for the endogenous RNA (ceRNA) network comprised 2 circRNAs, 154 miRNAs, and 6 mRNAs. KEGG analysis of these mRNAs included immune response-related pathways and cellular senescence.

Conclusions: The CeRNA regulatory network indicated that DE-circRNAs might participate in the processes of the cell immune response.

Key words: preeclampsia, circRNAs, expression profile, ceRNA, blood, immune response.

\section{Introduction}

Preeclampsia (PE) is a pregnancy-specific disorder associated with new-onset hypertension after 20 weeks of gestation and is one of the main causes of maternal and perinatal mortality and morbidity world-

\author{
Corresponding author: \\ Prof. Tao Wang \\ Key Laboratory of Birth \\ Defects and Related Diseases \\ of Women and Children \\ Center for Translational \\ Medicine \\ Sichuan University; \\ Department of Obstetrics \\ and Gynecology \\ Ministry of Education \\ West China Second \\ University Hospital \\ Sichuan University \\ Chengdu 610041, China \\ Phone/fax: 86-28-8550-3604 \\ E-mail: taowang@scu.edu.cn
}


wide, often accompanied by proteinuria or other organ damage such as to the kidney, brain or liver; it also may present in some women in the absence of proteinuria [1]. PE is classified into subtypes of early (delivered before 34 weeks) and late onset (delivered after 34 weeks) according to the time of diagnosis [2]. Many factors such as defective deep placentation, oxidative stress, endothelial dysfunction, and intravascular inflammation contribute to it [3], but the etiology and pathogenesis are far from being completely clarified. Among them, immune responses play a central role [4]. For example, NK cells interact with trophoblast cells and endothelial cells involved in remodeling the spiral arteries during early pregnancy [5]. In pregnant women with preeclampsia, the changes in circulating Tregs are reflected by high proportions of Th17 cells and the decreased IL-35 levels accompanied by elevated IL-17A levels [6].

Circular RNAs (circRNAs), defined as covalently closed circular RNAs, have recently been reported to be correlated with various pathological processes of cancer, diabetes, neurodegenerative disorders, etc. [7, 8]. Compared with other RNAs, the abundance of circRNAs is relatively low, but stability is high due to the circular configuration. Qian et al. first conducted a circRNA microarray and compared placentas from PE and healthy pregnancies and found 301 differentially expressed cirCRNAs that might be involved in the development of PE through acting as microRNA (miRNA) sponges [9]. Subsequently, an increasing number of studies have demonstrated the involvement of circRNAs in the pathogenesis of PE using high-throughput sequencing and bioinformatics techniques [10, 11]. Still, it cannot be ruled out that some novel circRNAs may be missed due to tissue-specific characteristics and database updates.

CircRNAs can interact with other RNAs and proteins, acting as miRNA sponges to regulate the transcription; they are also translated into pro- teins in rare cases [12]. Also, the miRNA response elements shared by circRNA and mRNA competitively bind to miRNAs, thereby influencing the regulatory function of miRNAs and gene expression [13]. Studies often focus on competing for en dogenous RNAs (ceRNAs) such as long non-coding RNA, circRNAs, and mRNAs to understand the pathogenesis of diseases [14, 15].

In this study, the differential expression profiles of circRNAs in healthy and PE pregnant women were demonstrated by high-throughput sequencing and predicted the potential functions by gene ontology (GO) and KEGG pathway analyses. Moreover, the differential expression mRNAs and the predicted miRNAs targeted by circRNAs were gained to generate a circRNA-miRNA-mRNA network. This study aims to use the ceRNA network to strengthen the understanding of the pathogenesis of PE and reveal the possible molecular mechanisms.

\section{Material and methods}

\section{Sample collection and RNA extraction}

A total of 47 pregnant women (30 cases with PE patients, including 10 with early-onset PE and 20 with late-onset PE, and 17 "healthy" pregnant women without hypertension) from the West China Second University Hospital, Sichuan University, were enrolled. The samples were collected from October 2021 to July 2021. All participants gave informed consent before participation. The Institutional Committee approved the study protocol for the Protection of Human Subjects (the Institutional Review Board of West China Second University Hos pital, Sichuan University, permit number 2019030).

As described in a previous study [16], the patients were diagnosed with PE according to the following criteria: new onset of hypertension after 20 weeks of gestation, systolic blood pressure (BP) $\geq 140 \mathrm{~mm} \mathrm{Hg}$ or diastolic $\mathrm{BP} \geq 90 \mathrm{~mm} \mathrm{Hg}$ on two oc-

Table I. Clinical characteristics of the study subjects

\begin{tabular}{|c|c|c|c|}
\hline Characteristics & $\begin{array}{l}\text { Normal term pregnancy } \\
\qquad(n=17)\end{array}$ & $\begin{array}{l}\text { Early onset preeclampsia } \\
\qquad(n=10)\end{array}$ & $\begin{array}{l}\text { Late onset preeclampsia } \\
\qquad(n=20)\end{array}$ \\
\hline Age [years] & $32.35 \pm 1.03(23-39)$ & $31.9 \pm 0.79(27-36)$ & $32.75 \pm 1.87(27-41)$ \\
\hline BMI $\left[\mathrm{kg} / \mathrm{m}^{2}\right]$ & $26.57 \pm 0.61(22.31-30.67)$ & $29.95 \pm 1.61(20.83-36.33)^{*}$ & $30.51 \pm 1.87(24.46-36.33)^{\star *}$ \\
\hline Primigravida & $8(47.1 \%)$ & $6(60.0 \%)$ & $14(70.0 \%)$ \\
\hline $\begin{array}{l}\text { Gestational age at delivery } \\
\text { [weeks] }\end{array}$ & $38.99 \pm 0.27(37-41)$ & $30.06 \pm 4.77(14.14-33.71)^{\star *}$ & $36.31 \pm 2(34.14-40.86)^{\star *}$ \\
\hline $\begin{array}{l}\text { Systolic blood pressure } \\
{[\mathrm{mm} \mathrm{Hg}]}\end{array}$ & $117.06 \pm 1.19(107-119)$ & $155.6 \pm 16.94(122-165)^{\star *}$ & $151.4 \pm 8.6(126-170)^{*}$ \\
\hline $\begin{array}{l}\text { Diastolic blood pressure } \\
{[\mathrm{mm} \mathrm{Hg}]}\end{array}$ & $74 \pm 1.56(61-88)$ & $98.9 \pm 11.63(75-114)^{\star \star}$ & $98.2 \pm 5.76(78-121)^{\star \star}$ \\
\hline 24-h urinary protein [g] & - & $2.71 \pm 2.73(0.357-7.903)^{\star \star}$ & $1.32 \pm 0.45(0.3-4.723)^{\star *}$ \\
\hline
\end{tabular}


casions at least 4 hours apart, 24-hour proteinuria $\geq 0.3 \mathrm{~g}$. According to the gestational age at diagnosis and/or delivery, PE has been classified into early-onset $\mathrm{PE}(<34$ gestational weeks) and late-onset $P E$ ( $>34$ gestational weeks). Pregnant women without PE were included as controls. In control subjects the normal blood pressure measurements were taken on the day of enrollment. Exclusion criteria included multiple pregnancies and transplanted organs, other pregnancy complications (e.g., chronic hypertension and diabetes mellitus), other complications (e.g., renal diseases, oncological diseases and autoimmune diseases), and any known fetal anomalies. The clinical characteristics are shown in Table I. Before delivery, the whole blood samples (3.0-5.0 ml) were collected in a sterile EDTA-containing vacutainer tube and cut carefully into pieces. Then, we froze samples at $-80^{\circ} \mathrm{C}$ immediately after adding $1 \mathrm{ml}$ of TRIzol reagent.

\section{RNA extraction and sequencing}

Five pairs of samples were randomly selected for RNA extraction and sequencing. Total RNA was extracted using the mirVana miRNA Isolation Kit following its protocol and quantified with a NanoDrop ND 2000 spectrophotometer. According to the manufacturer's instructions, the libraries were constructed using TruSeq Stranded Total RNA with Ribo-Zero Gold. Then these libraries were sequenced on the Illumina sequencing platform (HiSeq 2500 or other platforms), and $150 \mathrm{bp} /$ 125 bp paired-end reads were generated.

\section{Identification and quantification of human circRNAs}

We first used sortmerna software to remove the residual rRNA [17], then conducted quality testing through FASTQC software to ensure that high-quality reads [18] that have been filtered and tested strictly, called clean reads, were obtained, and the expression patterns of circRNAs and mRNAs were obtained. CIRI software, which is the authoritative software for circRNA prediction, is highly sensitive and can reduce false positives through multiple screening [19]. Matched to the circBase and CIRCpedia database, CIRI got Single Circ-Bed after predicting and merging samples and then counted the number of junctions reads of each predicted circRNA in different samples. DESeq software was applied to analyze differential expression among the samples and to set the fold-change of $\geq 2$, and $p$-values of $<0.05$ were identified as significantly differentially expressed transcripts. All subsequent bioinformatics analyses were from these five pair samples.

\section{Real-time quantitative PCR assay}

Total RNA was extracted for 30 PE samples and 17 control samples without PE, and was subjected to cDNA synthesis using the Prime Script RT reagent Kit (Takara, Dalian, China) following the manufacturer's instructions. Real-time quantitative PCR (RT-qPCR) was performed in the Light Cycle 480 Real-Time PCR Detection System (Roche, Germany) using qPCR SYBR Green Master (Vazyme, Nanjing, China). Referring to the other study [20], four upregulated and two downregulated circRNAs (Table II) were selected based on the fold change value $>2, p$-value $<0.05$, and expression levels for validation by qRT-PCR. The divergent primer pairs were designed using circPrimer software and Primer 5.0 for target circRNAs; the primer sequences used are shown in Table I. Relative expression of circRNAs was analyzed using the $2^{\Delta \Delta \mathrm{Ct}}$ method with normalization to $\beta$-actin expression and used as a quantitative control.

\section{Functional enrichment analysis}

The differentially expressed circRNAs were subjected to GO to illustrate the genetic regulatory networks according to the cellular component, biological process, and molecular function aspects (http://www.geneontology.org) [21]. Likewise, KOBAS software (KEGG Orthology-Based Annotation System) was used to conduct a KEGG pathway enrichment analysis of the networks [22].

Table II. Divergent primers of circRNAs for quantitative real-time PCR

\begin{tabular}{|lcc|}
\hline circBase ID & \multicolumn{1}{c|}{ Forward primer } & Reverse primer \\
\hline hsa_circ_0007717 & CAGGTCCAGAGGGAAGATGA & GGACTCTTGCTGGAACTTGC \\
\hline hsa_circ_0093055 & TTTTAGTCCCTCTGGCCTCA & CAATCCCTTTGTCCTTGAGC \\
\hline hsa_circ_0006460 & TGAGGGCTCCAGCTTGTATC & GATGTTGGCCTGGATGTTCTTG \\
\hline hsa_circ_0094867 & GACACCACCAAGGTCAAACA & CCTGAAATGCTGGGTCTGAT \\
\hline hsa_circ_0000048 & CAATCTCTGGGCACTGCATC & ACTCTGCGTGGGTGTAATGA \\
\hline hsa_circ_0009175 & CCACTGCAACATTACCTCCG & CTATACAGAGGCCAGGGACG \\
\hline$\beta$-actin & CATGTACGTTGCTATCCAGGC & CTCCTTAATGTCACGCACGAT \\
\hline The genome version is hg38. & &
\end{tabular}




\section{CeRNA network analysis}

Based on the validated DE-circRNAs verified by RT-qPCR, we further constructed a circRNA-miRNA -mRNA interaction network to explore the validated ceRNA mechanisms [23]. MiRNA-bound circRNAs can be identified using miRNA target gene prediction (miRanda database), on account of multiple miRNA binding sites of circRNAs. The threshold parameter was set as described previously: $S \geq 150, \Delta \mathrm{G} \leq-30 \mathrm{kcal} / \mathrm{mol}$ and strict $5^{\prime}$ seed pairing [24]. Subsequently, shared pairs of miRNA-mRNA and miRNA-circRNA were used to predict the ceRNA score using MuTaME [25] according to the following formula: ceRNA_score = MRE_for_share_miRNA/MRE_for_circRNA_miRNA, with the $p$-value being calculated using Benjamini and Hochberg's approach for controlling the false discovery rate. The positively correlated pairs of circRNA-mRNA based on ceRNA score principle were then considered as the true ceRNAs. Based on established co-expression data, deregulated ceRNA networks and circRNA or miRNA interactions of interest were mapped using Cytoscape software (V.3.7.1). The functions of these circRNAs were clarified according to the functional annotations of circRNA target mRNAs.

\section{Statistical analysis}

All statistical analysis was performed with SPSS 19 (IBM Corp.) and GraphPad Prism (Version 6.0, GraphPad Software, La Jolla, CA). The Kolmogorov-Smirnov test was used to evaluate the normal distribution of the data. The differences between two groups were evaluated by the independent $t$-test, and the nonparametric Mann-Whitney $t$-test was used for analysis when the data were not normally distributed. Data are shown as mean \pm SEM or interquartile range (IQR); $p<0.05$ was considered to indicate statistical significance. The diagnostic value of these DE-circRNAs was determined using receiver operating characteristics (ROC) curves.

\section{Results}

\section{Profiles of DE-circRNAs in the blood of women with PE and healthy pregnancy}

Compared with the information of human circRNAs in circBase (http://www.circbase.org/) utilizing sequence data, 688,310 circRNAs in the blood were identified. When matched to the circBase and CIRCpedia database, 25,886 circRNAs of these circRNAs were annotated, and 2722 circRNAs were newly discovered (Figure 1A). Analysis of high-throughput sequencing data revealed 139 DE-circRNAs between PE and healthy pregnant women. The results showed that 18 cir-
cRNAs were upregulated and 31 circRNAs were downregulated (fold change >1 and $p$-value $<0.05)$, as shown in heat maps and volcano plots (Figures 1B, 1C).

\section{Functional analysis of DE-circRNAs}

Gene ontology annotation analysis and the KEGG pathway were used to gain insights into the functions of DE-circRNAs. As shown in Figure 2A, enriched GO terms of the vital PE-related circRNAs were presented according to biological processes, cellular components, and molecular functions. For biological processes, DE-circRNAs were markedly enriched in terms such as biological regulation, immune system process, metabolic process, and so on. For molecular function, the vital PE-related circRNAs were mainly associated with binding, catalytic activity, and protein binding transcription factor activity. Meanwhile, the most enriched KEGG pathways included transport and catabolism, membrane transport, cell growth and death. The human diseases enrichment result shows that they might be involved in infectious disease, cancer, cardiovascular disease, etc. (Figure 2B). Among them, cardiovascular complications are reported to be a common complication of PE [26].

\section{Validation of DE-circRNA expression by RT-qPCR}

Four upregulated and two downregulated circRNAs (Table I) were selected based on the highest expression, fold change value $>2$, and $p$-value $<0.05$ for validation by RT-qPCR with divergent primers. The results revealed that the expression levels of circRNAs (has-circ-0007717, hascirc-0006460, and has-circ-0093055) in the blood were significantly higher in both subtype groups of PE patients than those in healthy pregnant women; the differential expression levels of other circRNAs were not significant (Figures 3A-3F). Moreover, the expression levels of circRNAs did not differ significantly between the subtype groups. Then, the ROC curve analysis was performed to detect the diagnostic values of these DE-circRNAs for $P E$. The results of the area under the curve (AUC) showed that the values of has-circ-0007717, hascirc-0006460, and has-circ-0093055 were 0.64 $(p=0.11), 0.72(p=0.01)$, and $0.72(p=0.01)$, respectively (Figures 3G-3I).

The threshold that provided maximal sensitivity and specificity for the diagnosis of PE was determined as the cut-off value. As shown in Table III, the sensitivity and specificity of hsa_circ_0093055 were the highest, at $73.33 \%$ and $6 \overline{4} .71 \%$, respectively. Moreover, the positive predictive value and negative predictive value hsa_circ_0093055 were the highest, at $78.57 \%$ and $63.16 \%$, respectively. 
A

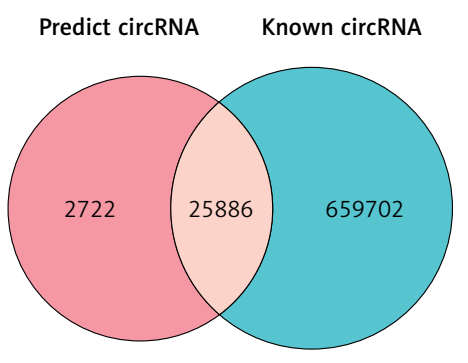

B
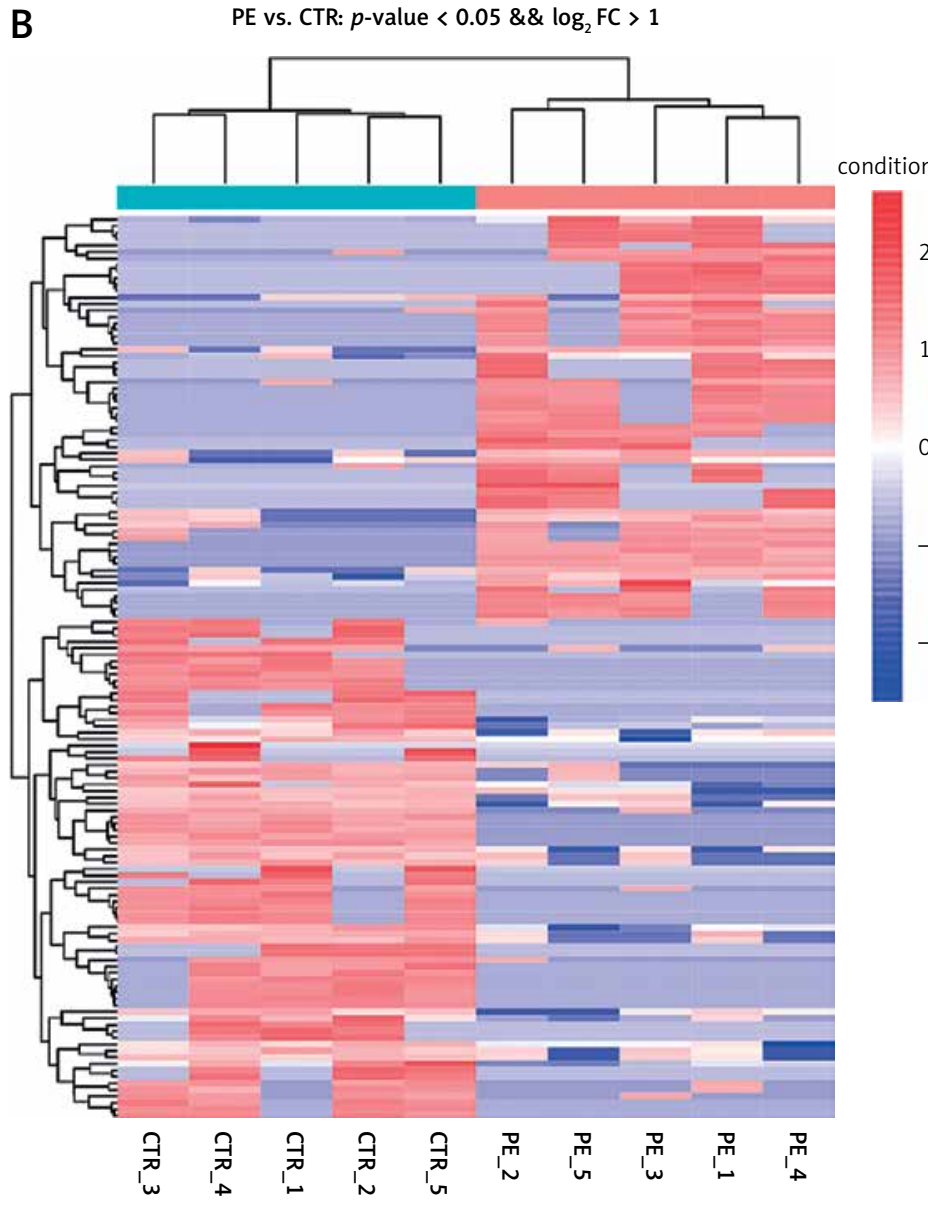

C

PE vs. CTR: $p$-value $<0.05 \& \& \log 2 \mathrm{FC}>1$

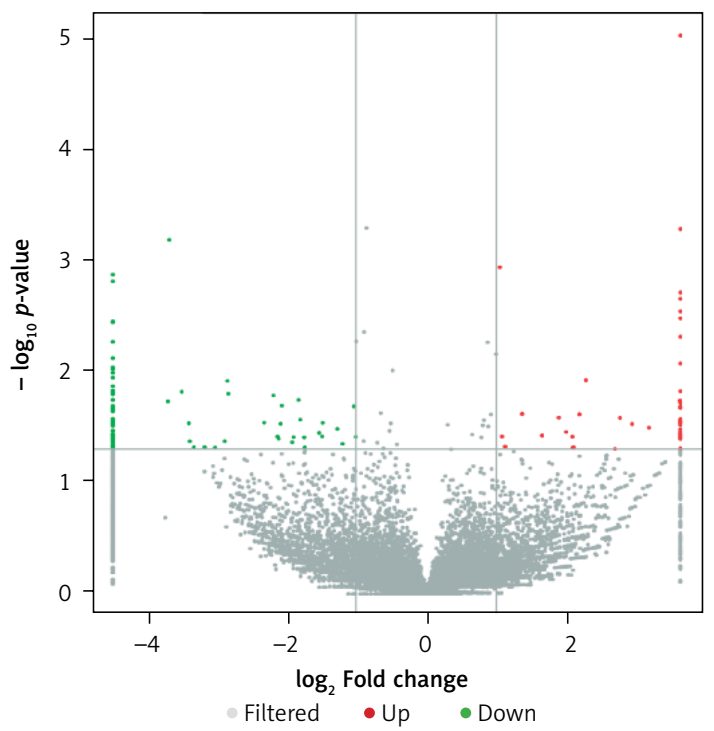

\section{CircRNA-miRNA-mRNA interaction analysis}

The three validated DE-circRNAs (hascirc-0007717, has-circ-0006460, and has-circ0093055) were chosen for further ceRNA network analysis. The miRanda algorithm was used to pre-
Figure 1. Differentially expressed (DE) circRNAs in the blood of women with preeclampsia (PE) and healthy pregnant women (CTR). A - The represented circRNAs were compared with the circRNAs in the circBase and CIRCpedia database. B - Heat map presentation of the expression profiles of the DE-circRNAs. Red reveals an increase and blue reveals a decrease in expression level when comparing PE with CTR. C - The volcano plot displays DE-circRNAs. Red dots indicate significantly up-regulated circRNAs, green dots indicate significantly down-regulated circRNAs, and gray dots indicate circRNAs with no significant difference

dict the binding between these miRNA-circRNA sequences, and a total of 2567 miRNA-circRNA relationship pairs were obtained; the top 100 of them were presented (Supplementary Figure S1A). Subsequently, we constructed the networks for circRNA-mRNA through co-expression analysis (Sup- 

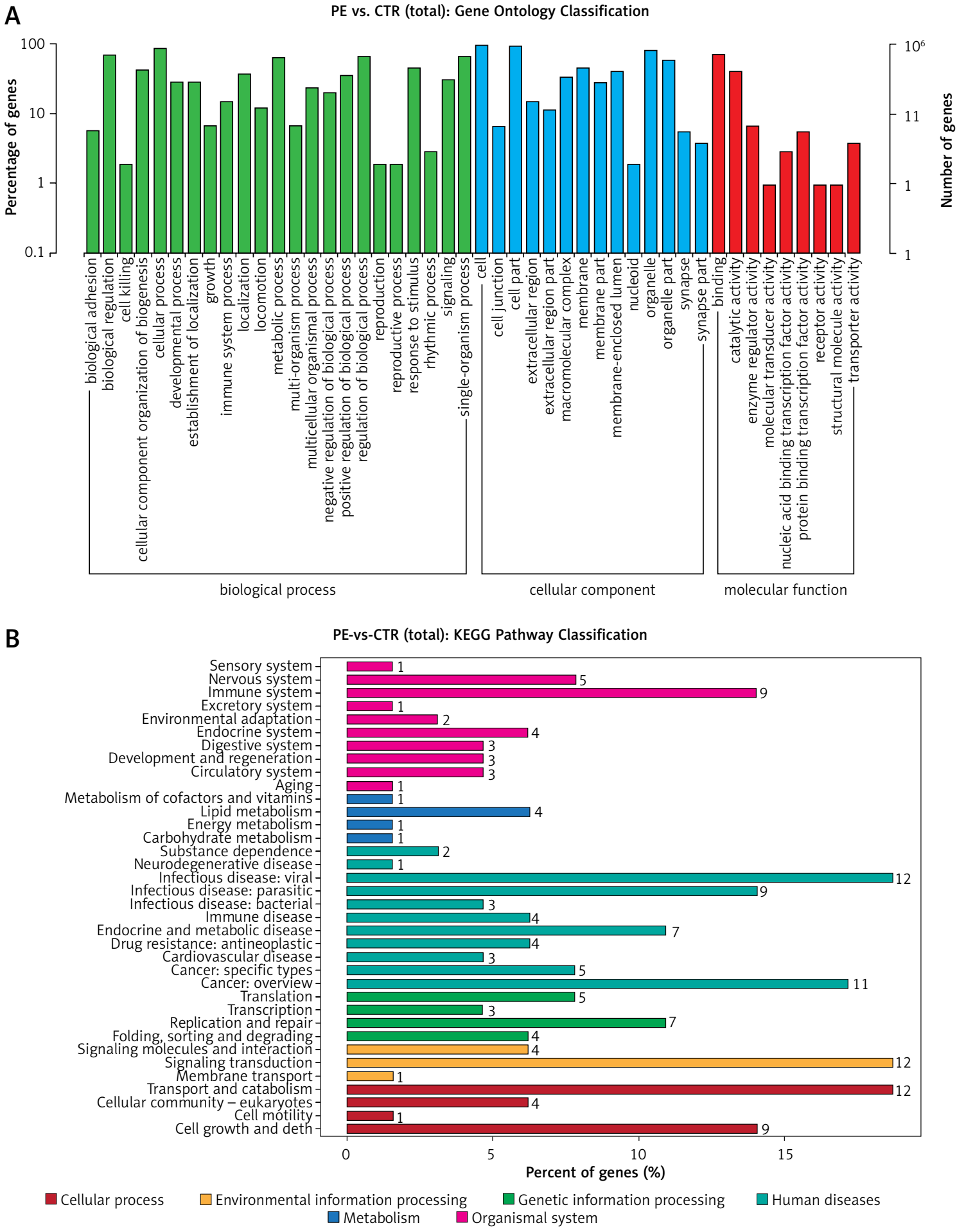

Figure 2. Functional analysis of differentially expressed (DE) circRNAs by gene ontology (GO) analysis and Kyoto Encyclopedia of Genes and Genomes (KEGG) pathway analyses. A - GO terms enriched in DE-circRNAs. B - KEGG pathways enriched in DE-circRNAs

$P E$ - preeclampsia, CTR-normal controls

plementary Figure S1B). As shown in Figure 4A, a global ceRNA network was constructed.

A hypergeometric distribution test was used to calculate the enrichment significance of these mRNAs regulated by circRNA in GO or KEGG entries as functional annotation of circRNA. For bio- logical processes, the GO terms were found to be markedly enriched in terms such as regulation of immune response, regulation of dendritic spine development, and negative regulation of tumor necrosis factor-mediated signaling pathway, and so on (Figure 4B). To get the pathways affected 
A

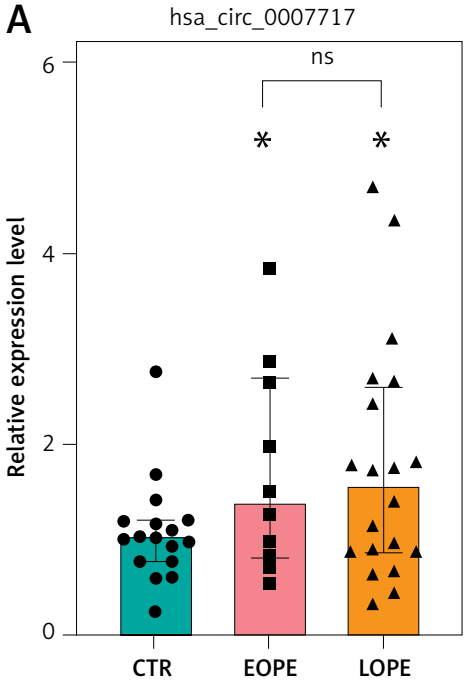

D

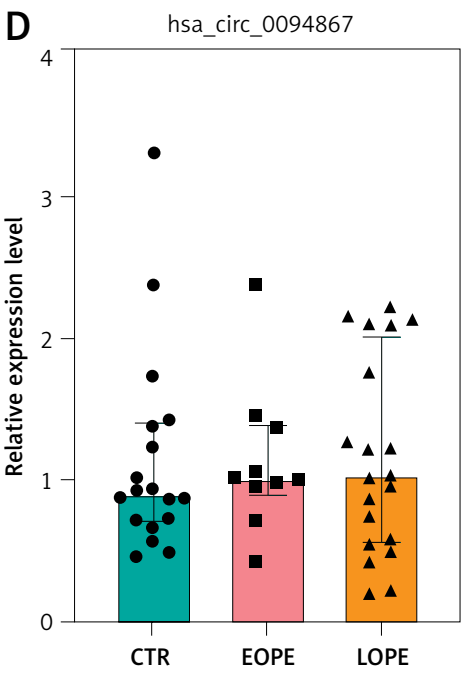

G

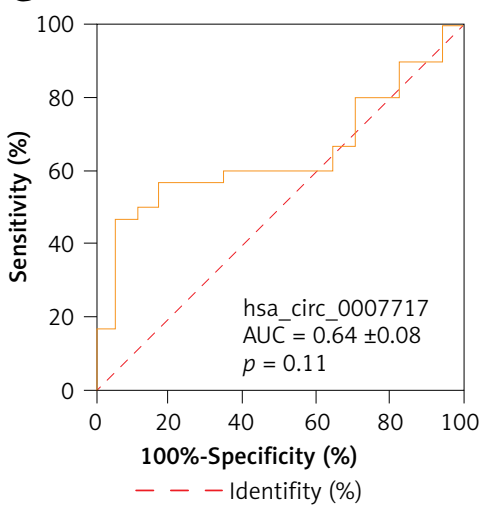

B

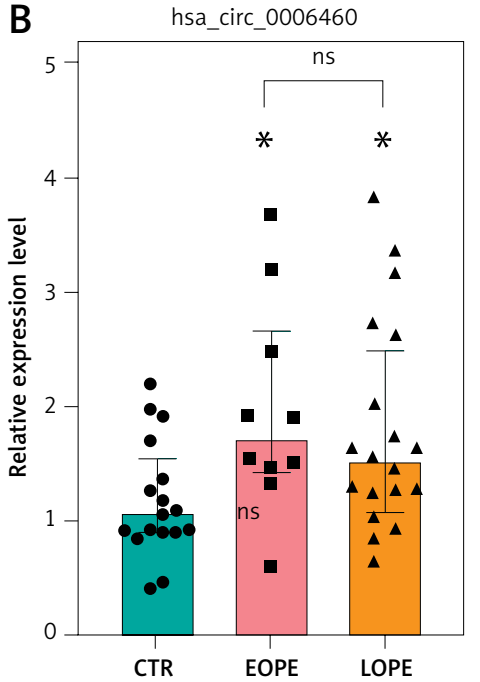

E

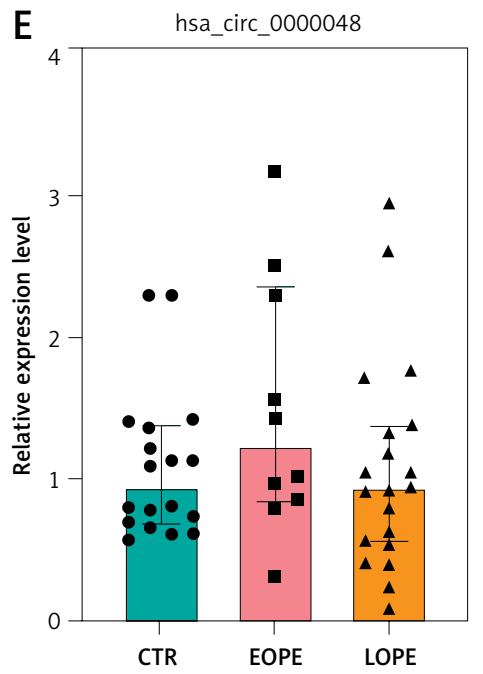

$\mathrm{H}$

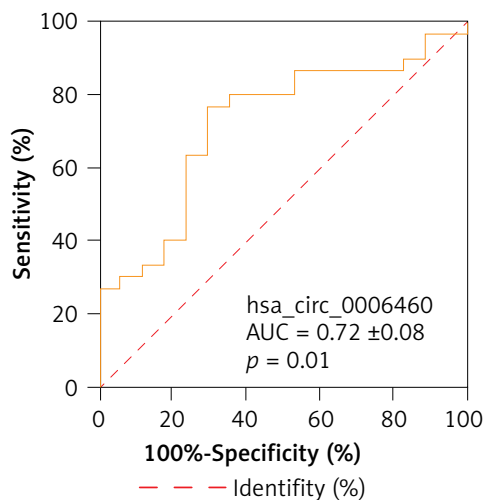

C hsa_circ_0093055

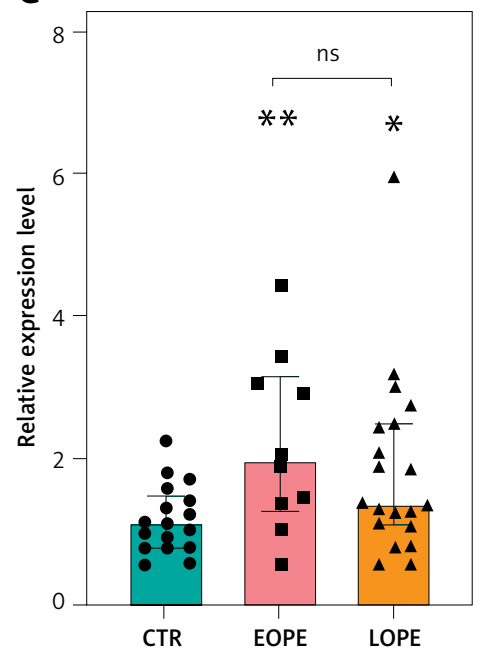

$\mathbf{F}$

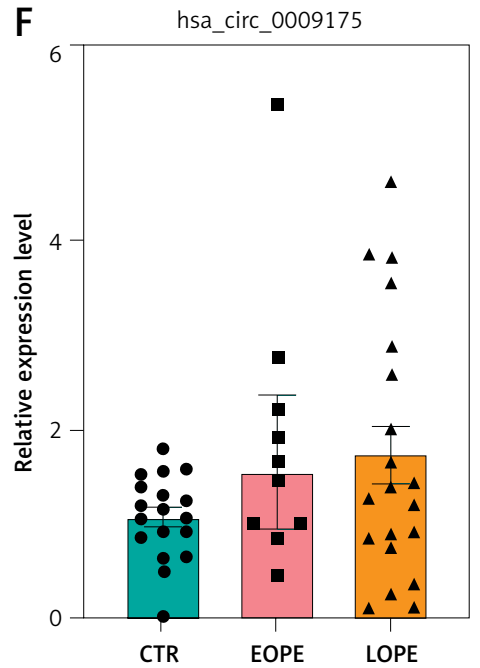

I

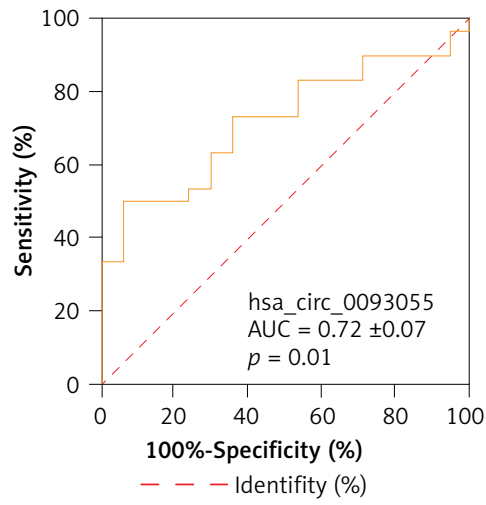

Figure 3. Relative expression of DE-circRNAs in blood from women with early-onset preeclampsia (EOPE), late-onset preeclampsia (LOPE), and normal pregnancy (CTR) detected by RT-qPCR. A-E - Relative expression of DE-circRNAs was determined by RT-qPCR. G-I - ROC analysis of PE patients based on has-circ-0007717, has-circ-0006460, and has-circ-0093055 expression. The presented values are the means \pm SEM or interquartile range (IQR), ${ }^{*} p<0.05$, ${ }^{* *} p<0.01, \mathrm{NS}-$ not significant 
Table III. Diagnostic value of validated DE-circRNAs for preeclampsia

\begin{tabular}{|lccc|}
\hline Parameter & hsa_circ_0007717 & hsa_circ_0093055 & hsa_circ_0006460 \\
\hline Sensitivity (Se) (\%) & 56.67 & 73.33 & 76.67 \\
\hline Specificity (Sp) (\%) & 82.35 & 64.71 & 70.59 \\
\hline Positive predictive value (PPV) (\%) & 85 & 78.57 & 73.33 \\
\hline Negative predictive value (NPV) (\%) & 51.85 & 63.16 & 52.94 \\
\hline
\end{tabular}

by our validated circRNAs, we performed a KEGG analysis of the six mRNAs in the network. The result indicated that antigen processing and presentation, natural killer cell-mediated cytotoxicity, and cellular senescence might be involved and have been reported to participate in the occurrence of $\mathrm{PE}$ (Figure $4 \mathrm{C}$ ).

\section{Discussion}

It is well accepted that circRNAs are involved in many obstetric complications by function prediction, including fetal growth restriction and infection during pregnancy [27]. Also, a few researchers have focused on the relationship between circRNAs and PE. Studies revealed the expression profile of cirCRNAs and their potential regulatory targets in the placentas of PE [10, 28, 29]. For example, a total of 180 DE-circRNAs were detected; hsa_circ_0011460 may serve as a potential therapeutic target for patients with PE with severe features [29]. Tong et al. conducted an RNA sequencing analysis to compare gene expression between the decidua of PE and normal pregnancy and found 293 key PE-related genes [30]. However, most of these studies were carried out in the placental tissue of PE. Fewer studies involve analysis of peripheral blood samples from PE patients to explore the related pathological mechanisms. In this study, we performed high-throughput sequencing to discover 139 DE-circRNAs in the circulation from PE and normal controls; their biological functions and the possible mechanism of PE were predicted by GO and KEGG analyses. DE-circRNAs were found to be markedly enriched in biological processes such as biological regulation, immune system processes, metabolic processes and so on. Meanwhile, the human diseases enrichment result shows that they might be involved in infectious disease, cancer, cardiovascular disease, etc. Cardiovascular complications are known to usually be associated with PE. These observations suggested that DE-circRNAs may be involved in the pathogenesis of PE.

Six circRNAs were selected to confirm the results of the RNA sequencing by RT-qPCR. Consistently with the sequencing results, the expression of these circRNAs (hsa_circ_0007717, hsa_circ_0006460, and hsa circ 0093055) was significantly increased in PE. It indicates that our sequencing data can truly reflect the expression of genes in the blood of PE patients. Moreover, the expression levels of circRNAs were not affected by gestational weeks at delivery and were closely associated with this disease. Ping et al. constructed a ceRNAs network based on all DE-circRNAs and determined that hsa_ circRNA 13301 was the only upregulated circRNA in ceRNA being targeted by four miRNAs in the blood of PE patients [11]. Distinctively, we selected the three validated circRNAs to perform further circRNA-miRNA -mRNA interaction networks and bioinformatics analysis. It is worth noting that the GO terms were enriched in the immune response and negative regulation of the tumor necrosis factor (TNF)-mediated signaling pathway, and the KEGG analysis showed that the antigen processing and presentation and natural killer (NK) cell-mediated cytotoxicity might be involved in the occurrence of PE. As we know, maternal adaptation to the fetus (paternal alloantigens) is vital in the embryonic implantation stage. During a normal pregnancy, immune cells either assist in required gestational processes or show immunoregulatory roles and are partially inactivated [31]. The critical role of NK cells in the pathogenesis of PE has been shown in numerous studies. Compared to healthy pregnancies, lower numbers and functional changes of NK cells were found in pregnant women with PE [32, 33]. Generally, natural cytotoxicity receptors (NCRs) are the major receptors involved in NK cytotoxicity and function in the recognition and lysis of tumor cells by NK cells. A study reported that pregnant women with PE present immunological abnormalities of NCRs on peripheral blood NK cells during pregnancy [34]. In terms of NK cell cytokine production, the decreased expression of NKP46 ${ }^{+}$NK cells in women with PE may be responsible for the high production of TNF- $\alpha$ in women with PE [35]. TNF- $\alpha$ blockade improves NK cell activation, hypertension, and mitochondrial oxidative stress in a preeclamptic rat model [36]. Overall, has-circ-0006460 and has-circ-0093055 may trigger a disorder in the maternal immunological response in pregnant women with PE.

The CeRNA hypothesis firstly proposed that regulatory networks working predict and manipulate through miRNA competition [37]. The expression of mRNA and post-transcriptional gene control are regulated by circRNAs [38]; thus, the functional analysis of circRNA-targeted mRNAs may help us deeply understand the role of circRNAs in the pathogenesis of PE. Hence, the function of these 


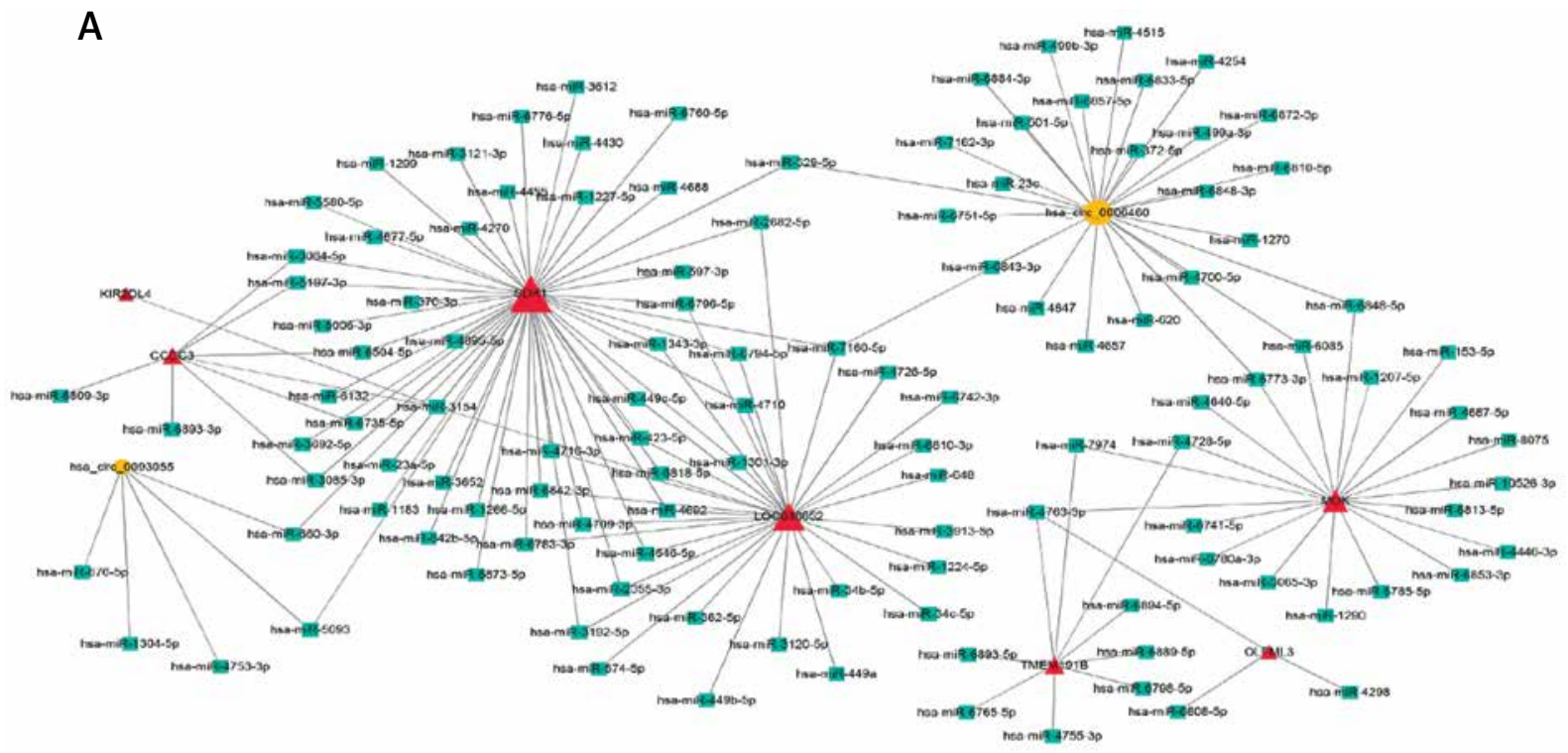

B

Statistic of GO enrichment

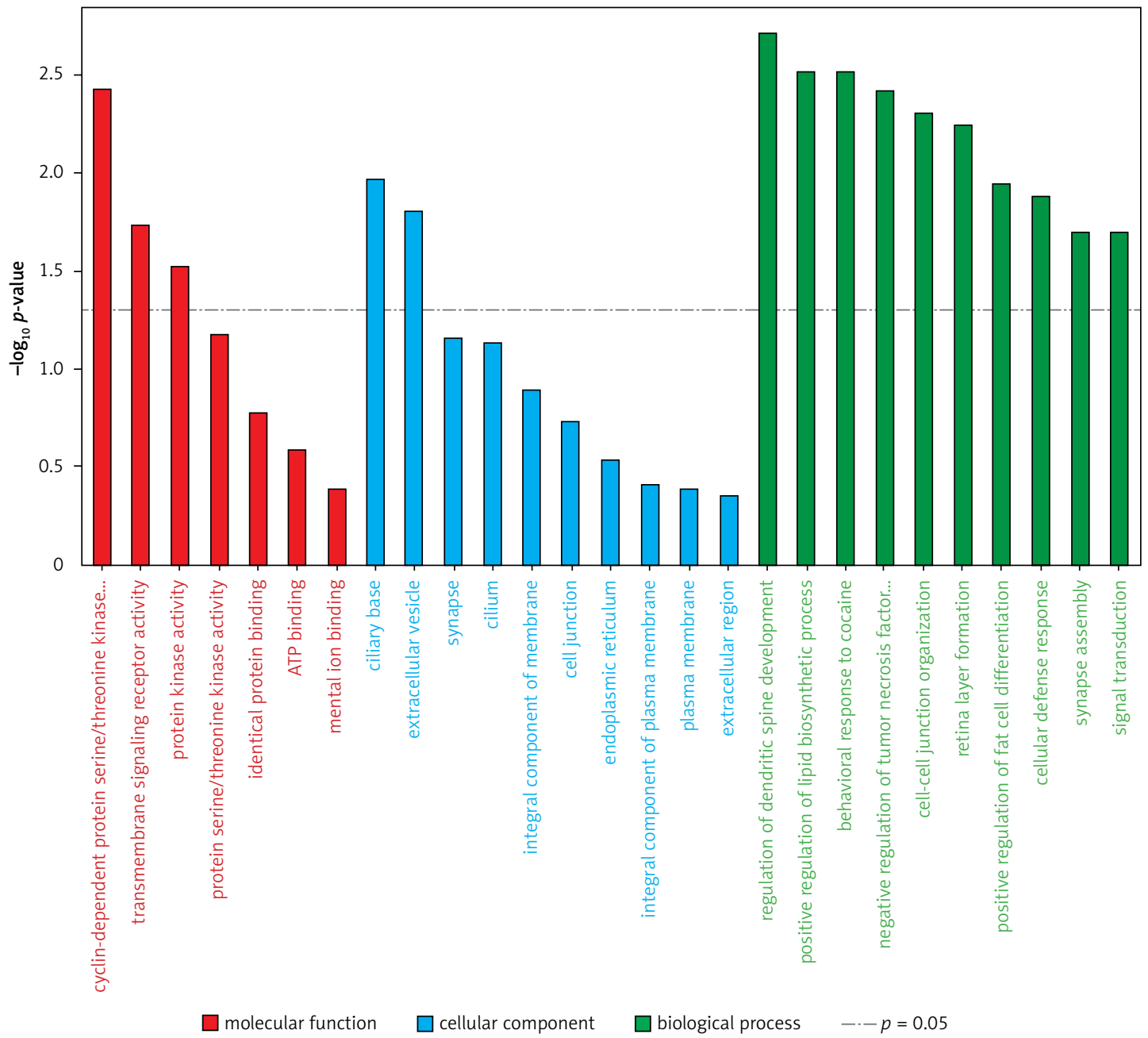

Figure 4. CeRNA network and functional analysis of validated DE-circRNA. A - The ceRNA network was based on circRNA/miRNA and miRNA/mRNA interactions. This ceRNA network includes has-circ-0007717, has-circ-0006460, and has-circ-0093055. The edges represent sequence matching, and circRNAs connect the expression of mRNAs through miRNAs. Round nodes (orange) represent circRNAs, quadrate nodes (light green) represent miRNAs, and triangular nodes (red) represent correlated mRNAs. The size of the node indicates the number of interactions. B, C-GO terms and top 30 KEGG pathways enriched in the mRNAs regulated by circRNAs 
C Top 30 of enrichment

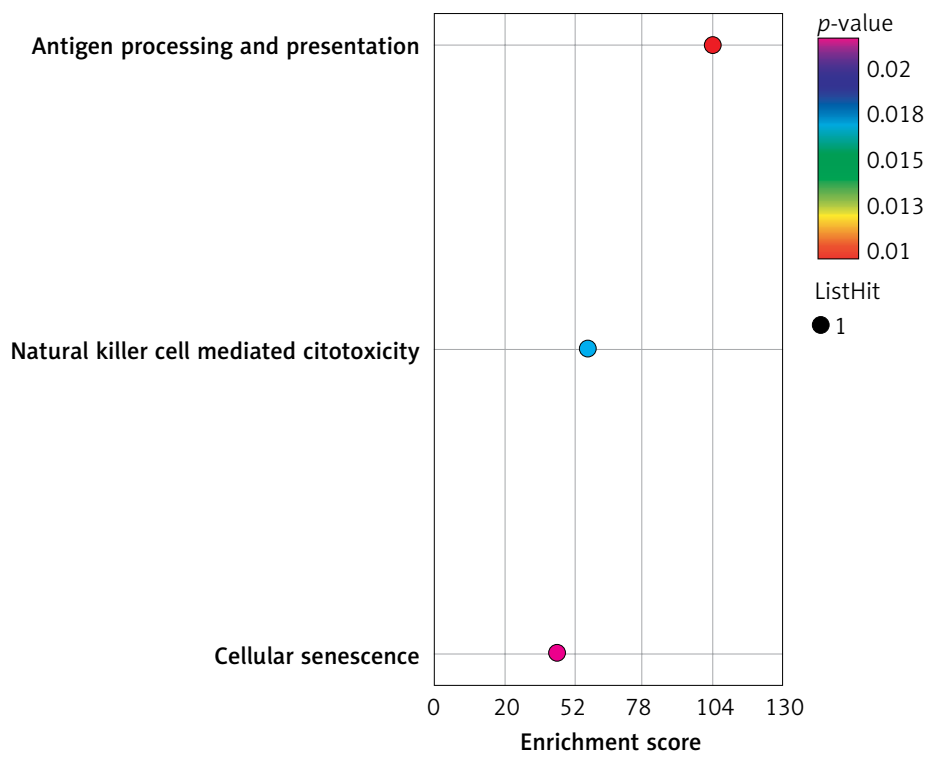

Figure 4. Cont. C - GO terms and top 30 KEGG pathways enriched in the mRNAs regulated by circRNAs

six mRNAs in the network was analyzed. As a newly identified secretory protein, the coiledcoil domain containing 3 (CCDC3) is expressed in adipose tissues and vascular endothelial cells. It was reported that $\mathrm{CCDC} 3$ represses the TNF- $\alpha$-induced pro-inflammatory response in endothelial cells [39], and this process is closely related to the development of PE. KIR2DL4 is the framework of killer cell immunoglobulin-like receptors (KIRs) that implicate the interaction between $\mathrm{dNK}$ and extravillous trophoblasts in vascular remodeling and its higher expression induces a higher risk of PE [40]. Similarly, SDK 1 and OLFML3 also reflect immune function $[41,42]$. MOK belongs to the MAP kinase superfamily used to treat various inflammatory conditions [43]. Therefore, these mRNAs further indicate that has-circ-0006460 and hascirc-0093055 could mediate the occurrence of PE through improper immune regulation.

There are several limitations to the study. (1) The characteristic features of pregnant women are different in these groups, not homogeneous. The expression of genes may be influenced by gestational age, especially. Hence, validation with a larger sample size may contribute to improving the power of the results and supporting the use of these circRNAs (hsa_circ_0007717, hsa circ_0006460, and hsa_circ_0093055) for finding the molecular mechanisms that drive the progression of PE. (2) Possible mechanisms for these cirCRNAs were only predicted using bioinformatics; further research for functional verification and investigation of downstream signaling molecules would be performed in the subsequent study. (3) The diagnostic value of hsa_circ_0093055 did not show excellent results, which needs to be confirmed by a follow-up study with larger sample sizes.

In conclusion, this study demonstrates the existence of dysregulated circRNAs in the blood of pregnant women with PE and healthy pregnant women. The higher expression levels of circRNAs (has-circ-0007717, has-circ-0006460, and hascirc-0093055) were verified by RT-qPCR. Then, we focused on these circRNAs to perform circRNA-miRNA-mRNA interaction network and bioinformatic analysis. The results revealed that has-circ-0006460 and has-circ-0093055 might trigger a disorder in the maternal immunological response in pregnant women with PE. This study provides profiles of circular RNAs in human circulation and new insights into the pathogenesis of PE. It is of great significance to find the molecular mechanisms that drive the progression of $\mathrm{PE}$, which may lead to more effective clinical therapeutic schedules.

\section{Acknowledgments}

This study was financially supported by grants from the National Natural Science Foundation of China (No. 81871188).

\section{Conflict of interest}

The authors declare no conflict of interest.

References

1. Mol BWJ, Roberts CT, Thangaratinam S, Magee LA, de Groot CJM, Hofmeyr GJ. Pre-eclampsia. Lancet 2016; 387: 999-1011.

2. ACOG Committee members and authors. Gestational hypertension and preeclampsia: ACOG Practice Bulletin, Number 222. Obstet Gynecol 2020; 135: e237-60. 
3. Rana S, Lemoine E, Granger JP, Karumanchi SA. Preeclampsia: pathophysiology, challenges, and perspectives. Circ Res 2019; 124: 1094-112.

4. Miller D, Motomura K, Galaz J, et al. Cellular immune responses in the pathophysiology of preeclampsia. J Leukoc Biol 2022; 111: 237-60.

5. Ratsep MT, Felker AM, Kay VR, Tolusso L, Hofmann AP, Croy BA. Uterine natural killer cells: supervisors of vasculature construction in early decidua basalis. Reproduction 2015; 149: R91-102.

6. Cao W, Wang X, Chen T, et al. The expression of Notch/ Notch ligand, IL-35, IL-17, and Th17/Treg in preeclampsia. Dis Markers 2015; 2015: 316182.

7. Jeck WR, Sharpless NE. Detecting and characterizing circular RNAs. Nat Biotechnol 2014; 32: 453-61.

8. Memczak S, Jens M, Elefsinioti A, et al. Circular RNAs are a large class of animal RNAs with regulatory potency. Nature 2013; 495: 333-8.

9. Qian Y, Lu Y, Rui C, Qian Y, Cai M, Jia R. Potential significance of circular RNA in human placental tissue for patients with preeclampsia. Cell Physiol Biochem 2016 39: 1380-90.

10. Zhou W, Wang $H, W u X$, et al. The profile analysis of circular RNAs in human placenta of preeclampsia. Exp Biol Med (Maywood) 2018; 243: 1109-17.

11. Ping Z, Ai L, Shen H, Zhang X, Jiang H, Song Y. Identification and comparison of circular RNAs in preeclampsia. Peerl 2021; 9: e11299.

12. Ashwal-Fluss R, Meyer M, Pamudurti NR, et al. CircRNA biogenesis competes with pre-mRNA splicing. Mol Cell 2014; 56: 55-66.

13. Han Q, Zhang R, Ma L, Shao L, Feng M. CircHIPK3 knockdown suppresses E2F3 expression to inhibit the viability and promote the apoptosis of lens epithelia cells in age-related cataract by sponging miR-499a-5p. Arch Med Sci 2021; Manuscripts accepted: https://doi. org/10.5114/aoms/138262.

14. Wang Z, Gu J, Han T, Li K. High-throughput sequencing profile of laryngeal cancers: analysis of co-expression and competing endogenous RNA networks of circular RNAs, long non-coding RNAs, and messenger RNAs. Ann Transl Med 2021; 9: 483.

15. Shi Y, Song R, Wang Z, et al. Potential clinical value of circular RNAs as peripheral biomarkers for the diagnosis and treatment of major depressive disorder. EBioMedicine 2021; 66: 103337.

16. Hypertension in pregnancy. Report of the American College of Obstetricians and Gynecologists' Task Force on Hypertension in Pregnancy. Obstet Gynecol 2013; 122: 1122-31.

17. Kopylova E, Noe L, Touzet H. SortMeRNA: fast and accurate filtering of ribosomal RNAs in metatranscriptomic data. Bioinformatics 2012; 28: 3211-7.

18. Andrews S. FastQC: a quality control tool for high throughput sequence data 2010. https://www.bioinformatics.babraham.ac.uk/projects/fastqc/

19. Gao Y, Wang J, Zhao F. CIRI: an efficient and unbiased algorithm for de novo circular RNA identification. Genome Biol 2015; 16: 4

20. Brazert M, Kranc W, Celichowski P, et al. Novel markers of human ovarian granulosa cell differentiation toward osteoblast lineage: a microarray approach. Mol Med Rep 2019; 20: 4403-14.

21. Ashburner M, Ball CA, Blake JA, et al. Gene ontology: tool for the unification of biology. The Gene Ontology Consortium. Nat Genet 2000; 25: 25-9.

22. Kanehisa M, Goto S. KEGG: Kyoto Encyclopedia of Genes and Genomes. Nucleic Acids Res 2000; 28: 27-30.
23. Zhou JZ, Hu MR, Diao HL, Wang QW, Huang Q, Ge BJ. Comprehensive analysis of differentially expressed cirCRNAs revealed a ceRNA network in pancreatic ductaladenocarcinoma. Arch Med Sci 2019; 15: 979-91.

24. Zhao Y, Lin Q, Li N, et al. MicroRNAs profiles of Chinese Perch Brain (CPB) cells infected with Siniperca chuatsi rhabdovirus (SCRV). Fish Shellfish Immunol 2019; 84: 1075-82.

25. Tay Y, Kats L, Salmena L, et al. Coding-independent regulation of the tumor suppressor PTEN by competing endogenous mRNAs. Cell 2011; 147: 344-57.

26. Poniedzialek-Czajkowska E, Mierzynski R. Could vitamin $D$ be effective in prevention of preeclampsia? Nutrients 2021; 13: 3854

27. Maass PG, Glazar P, Memczak S, et al. A map of human circular RNAs in clinically relevant tissues. J Mol Med (Berl) 2017; 95: 1179-89.

28. Ou Y, Liu M, Zhu L, et al. The expression profile of circRNA and its potential regulatory targets in the placentas of severe pre-eclampsia. Taiwan J Obstet Gynecol 2019; 58: 769-77.

29. Deng N, Lei D, Huang J, Yang Z, Fan C, Wang S. Circular RNA expression profiling identifies hsa_circ_0011460 as a novel molecule in severe preeclampsia. Pregnancy Hypertens 2019; 17: 216-25.

30. Tong J, Zhao W, Lv H, Li WP, Chen ZJ, Zhang C. Transcriptomic profiling in human decidua of severe preeclampsia detected by RNA sequencing. J Cell Biochem 2018; 119: 607-15.

31. Aneman I, Pienaar D, Suvakov S, Simic TP, Garovic VD, McClements L. Mechanisms of key innate immune cells in early- and late-onset preeclampsia. Front Immunol 2020; 11: 1864.

32. Milosevic-Stevanovic J, Krstic M, Radovic-Janosevic D, Popovic J, Tasic M, Stojnev S. Number of decidual natural killer cells \& macrophages in pre-eclampsia. Indian J Med Res 2016; 144: 823-30.

33. Rabaglino MB, Post Uiterweer ED, Jeyabalan A, Hogge WA, Conrad KP. Bioinformatics approach reveals evidence for impaired endometrial maturation before and during early pregnancy in women who developed preeclampsia. Hypertension 2015; 65: 421-9.

34. Fukui A, Funamizu A, Yokota M, et al. Uterine and circulating natural killer cells and their roles in women with recurrent pregnancy loss, implantation failure and preeclampsia. J Reprod Immunol 2011; 90: 105-10.

35. Fukui A, Yokota M, Funamizu A, et al. Changes of NK cells in preeclampsia. Am J Reprod Immunol 2012; 67: 278-86.

36. Cunningham MW, Jayaram A, Deer E, et al. Tumor necrosis factor alpha (TNF-alpha) blockade improves natural killer cell (NK) activation, hypertension, and mitochondrial oxidative stress in a preclinical rat model of preeclampsia. Hypertens Pregnancy 2020; 39 : 399-404.

37. Salmena L, Poliseno L, Tay Y, Kats L, Pandolfi PP. A ceRNA hypothesis: the Rosetta Stone of a hidden RNA language? Cell 2011; 146: 353-8.

38. Kristensen LS, Hansen TB, Veno MT, Kjems J. Circular RNAs in cancer: opportunities and challenges in the field. Oncogene 2018; 37: 555-65.

39. Azad AK, Chakrabarti S, Xu Z, Davidge ST, Fu Y. () Coiledcoil domain containing 3 (CCDC3) represses tumor necrosis factor-alpha/nuclear factor kappaB-induced endothelial inflammation. Cell Signal 2014; 26: 2793-800.

40. Wallace AE, Whitley GS, Thilaganathan B, Cartwright JE. Decidual natural killer cell receptor expression is altered in pregnancies with impaired vascular remodeling and 
a higher risk of pre-eclampsia. J Leukoc Biol 2015; 97: 79-86.

41. Roberts AL, Gladish N, Gatev E, et al. Exposure to childhood abuse is associated with human sperm DNA methylation. Transl Psychiatry 2018; 8: 194.

42. Toedebusch RG, Lucchesi CA, Debebe ET, Wittenburg LA, Chen X, Toedebusch CM. Microglia-derived olfactomedin-like 3 promotes pro-tumorigenic microglial function and malignant features of glioma cells. Int J Mol Sci 2021; 22: 13052.

43. Hwang JH, Ma JN, Park JH, Jung HW, Park YK. Anti-inflammatory and antioxidant effects of MOK, a polyherbal extract, on lipopolysaccharidestimulated RAW 264.7 macrophages. Int J Mol Med 2019; 43: 26-36. 